\section{Amending Reduced Fish Meal Feeds with Phospholipids to Improve Performance of Hybrid Striped Bass}

\section{Abstract}

Objective: In carnivorous fishes like hybrid striped bass Morone chrysops female $x$ M. saxatilis male, sparing dietary fish meal with soybean meal may lead to reduced feed intake and growth performance. Dietary phospholipid supplements may help improve feed intake and growth. The present study was designed to test the effects of dietary supplementation with marine- or soy-origin lecithins (sources of phospholipids) on the growth performance of hybrid striped bass fed reduced fish meal, soybean meal-based feeds.

Methods: We evaluated the growth performance of hybrid striped bass fed diets containing $30 \%$ soybean meal or $10 \%$ fish meal $65 \%$ soybean meal with and without supplementation of soy- or marine-origin lecithin at 2 or $4 \%$ of the diet. Juvenile fish $(33 \pm 0.6 \mathrm{~g}$, mean $\pm \mathrm{SEM})$ were stocked in a recirculation aquaculture system ( 8 fish per tank), and diets were randomly assigned to tanks in quadruplicate $(n=4)$. Fish were fed assigned diets daily to apparent satiation for 9 weeks, after which standard metrics of growth performance were assessed.

Results: Growth performance was generally superior among fish fed the lecithinsupplemented diets. This effect was particularly evident among fish fed the $10 \%$ fish meal diet supplemented with 2 or $4 \%$ marine lecithin, which exhibited significantly greater feed intake, weight gain, and specific growth rate than those fed the $10 \%$ fish meal or $30 \%$ fish meal feed. Feed conversion ratio was not statistically different among the dietary treatments.

Conclusion: Our results suggest amending reduced fish meal feeds with phospholipids can improve feed intake and growth in hybrid striped bass, and supplementing the diet with marine lecithin in particular may increase hybrid striped bass growth beyond that typically achieved with fish meal-based formulations.

Keywords: Morone spp, sunshine bass, lecithin, fatty acid, phospholipid

\author{
Heidi A. Hill ${ }^{1}$, \\ Jesse T. Trushenski ${ }^{1,2}$, \\ Brian R. Gause' ${ }^{1}$ and \\ Jérôme Laporte ${ }^{1}$.
}

1. Center for Fisheries, Aquaculture, and Aquatic Sciences Southern Illinois University Carbondale, Carbondale, Illinois 62901-6511 USA.

2 Department of Fish and Game, Eagle Fish Health Laboratory, Idaho, Eagle, ID 83616 USA.

Corresponding author: esse T. Trushenski

巨; jesse.trushenski@idfg.idaho.gov

Tel: 208-939-2413

Fax: 208-939-2413.

Citation: Hill H, Trushenski J, Gause B, et al. Amending Reduced Fish Meal Feeds with Phospholipids to Improve Performance of Hybrid Striped Bass. J Anim Res Nutr. 2015 1:1.

Received: November 16, 2015, Accepted: December 09, 2015, Published: December 16, 2015

\section{Introduction}

The increased utilization of soybean meal in commercial aquafeeds has helped reduce fish meal dependence and made a significant contribution towards the development of costeffective and sustainable feeds for the fish farming sector [1]. Nonetheless, the extent to which fish meal can be spared by alternatives like soybean meal is still widely debated, and varies among taxa. In omnivorous fish diets, soybean meal typically allows for total replacement of fish meal without impacting the growth of the fish, whereas issues of palatability, digestibility or essential amino acid composition typically limit the performance of carnivorous fish when all or most of the fish meal fraction of the diet is replaced [1]. Although soy-derived proteins are welldigested by hybrid striped bass Morone chrysops female $\times M$. saxatilis male [2,3], various experiments have confirmed that soybean meal (amended with methionine supplements) could not fully replace fish meal without impairing specific growth rate 
and feed conversion ratio $[4,5,6]$. Often, impaired performance is linked to reduced intake of soybean meal-based feeds. The organoleptic profile of soybean meal is distinct from that of fish meal, and palatability factors (or the lack thereof) are commonly invoked to explain reduced acceptance of reduced or fish mealfree feeds by carnivorous fish. However, performance may also be influenced by the loss of nutrients present in fish meal but not soybean meal. Notably, fish meal is a rich source of phospholipids, with the lipid fraction ( $5-13 \%$, by weight) typically containing twice as much polar lipid as neutral lipid [7]. It is possible that supplementation with potentially beneficial, though perhaps not strictly essential phospholipids may improve intake and performance of fish fed reduced fish meal feeds.

The addition of phospholipids, in the form of lecithins, to aquafeeds has been shown to increase growth by increasing total feed intake in some fish. Feed intake and growth of rainbow trout Oncorhynchus mykiss [8,9], Japanese flounder Paralichthys olivaceus [10], amberjack Seriola dumerili [11] and Atlantic salmon Salmo salar $[12,13]$ were improved by dietary supplementation with soy lecithin. Although soy lecithin is more commonly used as a feed supplement, marine lecithin is also commercially available and may prove useful as a feed supplement. Marine lecithin, derived from fish, is high in 22:6n-3 (docosahexaenoic acid), an essential fatty acid for normal growth and development. While supplementation with lecithin may increase feed intake and growth performance, supplementation with essential fatty acids in the context of more 'potent' phospholipids (compared to neutral lipids) may further promote growth $[7,14,15]$. Little research has been undertaken to compare supplementation of marine lecithin with the more widely used soy lecithin. Accordingly, the present study was designed to test dietary supplementation with marine- or soy-origin phospholipids on the growth performance of hybrid striped bass fed reduced fish meal, soybean meal-based feeds.

\section{Methods}

Two practical feeds ( $40 \%$ protein, $14 \%$ lipid) previously evaluated in the Center for Fisheries, Aquaculture, and Aquatic Sciences (CFAAS, Carbondale, Illinois) [6] and formulated to be isolipidic/ isonitrogenous and to meet all known nutritional requirements of juvenile hybrid striped bass were slightly modified for use in the present work (Table 1). A feed containing approximately $30 \%$ menhaden fish meal (Special Select, Omega Protein, Houston, Texas) served as the control (30\% FM). A reduced fish meal feed was formulated to containing approximately $10 \%$ menhaden fish meal and soybean meal as the primary source of alternative protein (10\% FM). These two feeds previously yielded equivalent growth performance in hybrid striped bass [6]. Four experimental diets were derived from the $10 \% \mathrm{FM}$ formulation by supplementing the formulations with $2 \%$ or $4 \%$ supplemental phospholipids as marine lecithin (Marine Natural Lecithin LC 60, Phosphotech Laboratories, Saint-Herblain Cedex, France; $10 \% \mathrm{FM}+1 \mathrm{X}$ Marine $\mathrm{PL}, 10 \% \mathrm{FM}+2 \mathrm{X}$ Marine $\mathrm{PL}$ ) or soy lecithin (Yelkin ${ }^{\circledR}$ TS soy-derived lecithin, Archer Daniels Midland, Decatur, Illinois ; $10 \% \mathrm{FM}+1 \mathrm{X}$ Soy PL, $10 \% \mathrm{FM}+2 \mathrm{X}$ Soy $\mathrm{PL})$. The inclusion rates were calculated to represent one $(2 \%$ supplementation $=1 \mathrm{X})$ or two times $(4 \%$ supplementation
$=2 \mathrm{X}$ ) the estimated difference in phospholipid content between the $30 \% \mathrm{FM}$ and $10 \% \mathrm{FM}$ feeds, based on the estimated loss of fish meal-derived phospholipid and the phospholipid content of the lecithin products used. In other words, we calculated that reducing fish meal from approximately $30 \%$ to approximately $10 \%$ would result in the loss of approximately $1.2 \%$ phospholipid from the diet (assuming the fish meal contained 8-9\% total lipid and the lipid contained phospholipid and triglycerides in a ratio of $2: 1$ [7]), and thus $2 \%$ dietary supplementation with the lecithin products used was estimated to supply an equivalent amount of phospholipid (based on manufacturers' estimates, the lecithin products were assumed to contain $60 \%$ phospholipid). Lecithin supplements displaced an equivalent amount of menhaden fish oil in the formulations.

All ingredients were incorporated using a commercial-grade cutter mixer (Model CM450, Hobart Corporation, Troy, Ohio) and pelleted using an electric food grinder (Cabela's 1.5 HP, Cabela's, Sidney, Nebraska) fitted with a $4 \mathrm{~mm}$ die. Feeds were then dried to approximately $94 \%$ dry matter in a commercial grade food dehydrator (Harvest Saver R5-A, Commercial Dehydrator Systems Inc., Eugene, Oregon). Subsamples of feed were stored at $-80^{\circ} \mathrm{C}$ for subsequent analysis, whereas the remainder of the feed used during the trial was stored at $-20^{\circ} \mathrm{C}$. Each feed was analyzed in triplicate to determine proximate (Table 1) and fatty acid composition (Tables 2-4). All samples were first lyophilized (Freezone 6, Labconco, Kansas City, Missouri) to gravimetrically determine moisture content and then pulverized in preparation for further analysis. Ash content was determined gravimetrically after incineration in a muffle furnace at $650^{\circ} \mathrm{C}$ for 4 hours. Protein content was determined using a LECO protein analyzer (FP-528, LECO Corporation, St. Joseph, Michigan) according to the manufacturer's instructions. Total lipid content was determined gravimetrically following a modified chloroform/ methanol extraction from [16]. Fatty acid methyl esters (FAME) were obtained via acid-catalyzed transmethylation and identified/quantified via GC-FID (GC-17A, Shimadzu Corporation, Kyoto, Japan) as described by [17] (Table 2). Dietary lipid class composition was determined using procedures adapted from [18]. Briefly, crude lipid was extracted from feeds as before and resuspended in chloroform. Lipid classes were separated using 3- $\mathrm{mL}$ aminopropyl solid phase extraction columns (Supelclean ${ }^{\mathrm{TM}}$ LC-NH2 SPE Tubes, Supelco, Bellefonte, Pennsylvania) fitted to a Supelco VISIPREP ${ }^{\text {TM }}$ (Supelco, Bellfonte, Pennsylvania) vacuum separation unit. Neutral lipids were eluted using a 2:1 chloroform:2-proponal solution, and polar lipids were subsequently eluted using methanol. Fatty acid methyl esters were prepared, identified, and quantified in the same manner as those derived from total lipid samples (Tables 3, 4).

Juvenile hybrid striped bass $(33 \pm 0.6 \mathrm{~g}$, mean \pm SEM) were obtained from Keo Fish Farm (Keo, Arkansas) and stocked at 8 fish/tank into 24 tanks of a recirculation aquaculture system consisting of 150-L tanks with associated mechanical (bead filter) and biological (submerged media biofilter) filtration and supplemental aeration. Photoperiod was maintained at a 12:12 light/dark cycle, and temperature was maintained via ambient air temperature. Temperature and dissolved oxygen were monitored daily (YSI 550A, Yellow Springs Instruments, Yellow 
Table 1 Dietary formulation and proximate composition (values represent LS-means \pm SEM of triplicate samples).

\begin{tabular}{|c|c|c|c|c|c|c|}
\hline Ingredient (g/kg, as fed) & $30 \% \mathrm{FM}$ & $10 \% \mathrm{FM}$ & 10\% FM + 1X Marine PL & $10 \% \mathrm{FM}+2 \mathrm{X}$ Marine PL & $10 \%$ FM + 1 X Soy PL & $10 \% \mathrm{FM}+2 \mathrm{X}$ Soy $\mathrm{PL}$ \\
\hline Fish meal & 299.0 & 99.9 & 99.9 & 99.9 & 99.9 & 99.9 \\
\hline Soybean meal & 297.0 & 656 & 656 & 656.0 & 656 & 656.0 \\
\hline Wheat bran & 263.0 & 80.0 & 80.0 & 80.0 & 80.0 & 80.0 \\
\hline Canola oil & 50.8 & 37.4 & 37.4 & 37.4 & 37.4 & 37.4 \\
\hline Fish oil & 36.5 & 71.4 & 51.4 & 31.4 & 51.4 & 31.4 \\
\hline Carboxymethyl cellulose & 20.3 & 20.3 & 20.3 & 20.3 & 20.3 & 20.3 \\
\hline Dicalcium phosphate & 15.0 & 14.9 & 14.9 & 14.9 & 14.9 & 14.9 \\
\hline Sodium phosphate & 9.1 & 9.2 & 9.2 & 9.2 & 9.2 & 9.2 \\
\hline Choline & 5.5 & 5.5 & 5.5 & 5.5 & 5.5 & 5.5 \\
\hline Stay C (35\%) & 1.8 & 1.8 & 1.8 & 1.8 & 1.8 & 1.8 \\
\hline Vitamin premix ${ }^{a}$ & 1.1 & 1.1 & 1.1 & 1.1 & 1.1 & 1.1 \\
\hline Mineral premix ${ }^{b}$ & 0.9 & 0.9 & 0.9 & 0.9 & 0.9 & 0.9 \\
\hline DL-methionine & 0 & 1.6 & 1.6 & 1.6 & 1.6 & 1.6 \\
\hline Marine lecithin ${ }^{c}$ & 0 & 0 & 20.0 & 40.0 & 0 & 0 \\
\hline Soy lecithin ${ }^{d}$ & 0 & 0 & 0 & 0 & 20.0 & 40.0 \\
\hline \multicolumn{7}{|c|}{ Proximate composition (g/kg, dry matter basis except dry matter) } \\
\hline Dry matter & $950 \pm 0$ & $947 \pm 0$ & $935 \pm 2$ & $930 \pm 0$ & $939 \pm 0$ & $936 \pm 0$ \\
\hline Protein & $395 \pm 2$ & $392 \pm 3$ & $408 \pm 2$ & $414 \pm 0$ & $401 \pm 2$ & $410 \pm 3$ \\
\hline Lipid & $136 \pm 2$ & $137 \pm 2$ & $134 \pm 4$ & $126 \pm 0$ & $137 \pm 2$ & $134 \pm 2$ \\
\hline Ash & $67 \pm 9$ & $55 \pm 3$ & $62 \pm 3$ & $86 \pm 9$ & $56 \pm 4$ & $54 \pm 3$ \\
\hline
\end{tabular}

a Formulated to contain the following per $100 \mathrm{~g}$ of premix: $8.955 \mathrm{~g}$ cellulose, $8.3 \mathrm{~g}$ all-rac-alpha tocopheryl acetate, $12.5 \mathrm{~g}$ inositol, $25.0 \mathrm{~g}$ L-ascorbyl-2-polyphosphate, $12.5 \mathrm{~g}$ nicotinic acid, $6.25 \mathrm{~g}$ calcium pantothenate, $13.16 \mathrm{~g}$ menadione sodium bisulfate complex, $1.25 \mathrm{~g}$ thiamine mononitrate, $2.5 \mathrm{~g}$ pyridoxine hydrochloride, $7.5 \mathrm{~g}$ riboflavin, $1.0 \mathrm{~g}$ vitamin A palmitate, $0.45 \mathrm{~g}$ folic acid, $0.5 \mathrm{~g}$ cyanocobalamin, $0.01 \mathrm{~g}$ cholecalciferol, and $0.125 \mathrm{~g}$ biotin.

${ }^{b}$ Formulated to contain the following per $100 \mathrm{~g}$ of premix: $55.381 \mathrm{~g}$ cellulose, $24.897 \mathrm{~g}$ zinc oxide, $14.933 \mathrm{~g}$ ferrous sulfate, $3.47 \mathrm{~g}$ manganese oxide, $0.967 \mathrm{~g}$ cupric carbonate, $0.262 \mathrm{~g}$ potassium iodide, $0.06 \mathrm{~g}$ sodium selenate, and $0.03 \mathrm{~g}$ cobalt carbonate.

"Yelkin" TS soy-derived lecithin, Archer Daniels Midland, Decatur, Illinois.

${ }^{d}$ Marine Natural Lecithin LC 60, Phosphotech Laboratories, Saint-Herblain Cedex, France.

Springs, Ohio), whereas alkalinity, total ammonia-, nitrite-, and nitrate-nitrogen were quantified every 7-10 days. Water quality parameters were maintained within ranges suitable for hybrid striped bass culture [19] throughout the trial (temperature -24.3 $\pm 0.0{ }^{\circ} \mathrm{C}$; dissolved oxygen $-5.5 \pm 0.0 \mathrm{mg} / \mathrm{L}$; total ammonia $-0.1 \pm$ $0.1 \mathrm{mg} / \mathrm{L}$; nitrite-nitrogen $-0.0 \pm 0.0 \mathrm{mg} / \mathrm{L}$; nitrate-nitrogen -9.2 $\pm 0.9 \mathrm{mg} / \mathrm{L}$; alkalinity $-306.0 \pm 6.7 \mathrm{mg} / \mathrm{L}$ of $\mathrm{CaCO}_{3}$; mean $\pm \mathrm{SE}$ ). Dietary treatments were randomly assigned to quadruplicate tanks ( 4 tanks/treatment, $n=4$ ) and all fish were fed assigned diets once daily to apparent satiation. After 9 weeks of culture, fish were counted and group weighed by tank. Three fish were then randomly selected from each tank, sedated (bath immersion in a solution of tricaine methanesulfonate), euthanized by single cranial pithing, weighed, and dissected to remove and weigh liver and intraperitoneal fat tissues. Group and individual-based data were used, along with daily feeding records, to calculate production performance metrics as follows:

Weight Gain $(\%)=100 \times \frac{(\text { average final weight-average initial weight })}{\text { average initial weight }}$

Feed Conversion Ratio $(\mathrm{FCR})=\frac{\text { Cumulative Feed Intake }}{\text { average individual weight gain }}$ Specific growth rate (SGR, $\%$ body weight/day) $=100 \times \frac{\text { lnaverage final weight }- \text { lnaverage initial weight }}{\text { days of feeding }}$

Feed intake $(\%$ body weight/day $)=100 \times$

Cumulative Feed Intake initial individual weight $\times$ final individual weight $)^{0.5}$ days of feeding

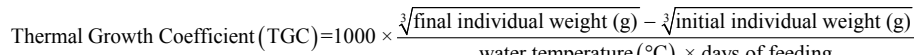

Hepatosomatic Index $(\mathrm{HSI})=100 \times \frac{\text { liver weight }}{\text { whole body weight }}$

Liposomatic Index $($ LSI $)=100 \times \frac{\text { intraperitoneal fat weight }}{\text { whole body weight }}$

Although multiple fish were sampled from each tank, replicate tanks served as the experimental units for all statistical analyses $(n=4)$. All growth performance data were analyzed by one-way analysis of variance (ANOVA, PROC GLIMMIX) using the Statistical Analysis System (version 9.3, SAS Institute, Cary, North Carolina) to determine the significance of dietary treatment effects. For parameters exhibiting significant treatment effects, Tukey's HSD tests were used for pairwise comparisons of means. For all statistical procedures, differences were considered significant at $\mathrm{p}<0.05$.

\section{Results}

Amending reduced fish meal hybrid striped bass feeds with marine or soy-derived phospholipids altered the composition of the feeds and the growth performance they yielded. Although proximate composition was relatively consistent among the feeds (Table 1), the diets were distinctly different with respect to total lipid FA composition (Table 2). These differences in fatty acid composition were particularly evident within the neutral 
Table 2 Fatty acid composition (\% fatty acid methyl esters [FAME]) of dietary total lipid. Values represent LS-means \pm SEM of triplicate samples."

\begin{tabular}{|c|c|c|c|c|c|c|}
\hline Fatty Acid(s) & $30 \%$ FM & $10 \% \mathrm{FM}$ & $10 \% \mathrm{FM}+1 \times$ Marine PL & 10\% FM + 2X Marine PL & $10 \% \mathrm{FM}+1 \mathrm{X}$ Soy $\mathrm{PL}$ & $10 \% \mathrm{FM}+2 \times$ Soy $\mathrm{PL}$ \\
\hline $14: 0$ & $4.2 \pm 0.0$ & $5.0 \pm 0.0$ & $4.4 \pm 0.0$ & $3.5 \pm 0.0$ & $4.0 \pm 0.0$ & $2.8 \pm 0.0$ \\
\hline $16: 0$ & $13.7 \pm 0.1$ & $14.6 \pm 0.1$ & $15.1 \pm 0.1$ & $14.9 \pm 0.1$ & $14.3 \pm 0.1$ & $13.8 \pm 0.1$ \\
\hline 18:0 & $3.3 \pm 0.0$ & $3.3 \pm 0.0$ & $3.3 \pm 0.0$ & $3.1 \pm 0.0$ & $3.4 \pm 0.1$ & $3.5 \pm 0.0$ \\
\hline SFA $^{a}$ & $22.5 \pm 0.2$ & $24.2 \pm 0.2$ & $24.1 \pm 0.2$ & $22.8 \pm 0.2$ & $22.8 \pm 0.1$ & $21.2 \pm 0.1$ \\
\hline $16: 1 n-7$ & $5.4 \pm 0.0$ & $6.6 \pm 0.0$ & $5.6 \pm 0.0$ & $4.3 \pm 0.0$ & $5.2 \pm 0.0$ & $3.7 \pm 0.0$ \\
\hline $18: 1 n-7$ & $3.0 \pm 0.0$ & $3.0 \pm 0.0$ & $2.9 \pm 0.0$ & $2.8 \pm 0.0$ & $2.8 \pm 0.0$ & $2.5 \pm 0.0$ \\
\hline $18: 1 n-9$ & $30.2 \pm 0.2$ & $24.4 \pm 0.2$ & $25.9 \pm 0.2$ & $27.6 \pm 0.2$ & $25.3 \pm 0.0$ & $27.3 \pm 0.1$ \\
\hline $20: 1 n-9$ & $0.9 \pm 0.0$ & $0.9 \pm 0.0$ & $1.1 \pm 0.0$ & $1.3 \pm 0.0$ & $0.8 \pm 0.0$ & $0.7 \pm 0.0$ \\
\hline MUFA $^{b}$ & $39.8 \pm 0.2$ & $35.3 \pm 0.3$ & $36.0 \pm 0.2$ & $36.3 \pm 0.2$ & $34.4 \pm 0.1$ & $34.5 \pm 0.2$ \\
\hline $18: 2 n-6$ & $14.0 \pm 0.0$ & $13.2 \pm 0.1$ & $13.5 \pm 0.0$ & $14.9 \pm 0.0$ & $19.6 \pm 0.0$ & $26.6 \pm 0.0$ \\
\hline $20: 4 n-6$ & $0.6 \pm 0.0$ & $0.7 \pm 0.0$ & $0.7 \pm 0.0$ & $0.7 \pm 0.0$ & $0.5 \pm 0.0$ & $0.4 \pm 0.0$ \\
\hline$n-6^{c}$ & $15.0 \pm 0.0$ & $14.4 \pm 0.0$ & $14.6 \pm 0.0$ & $15.9 \pm 0.0$ & $20.5 \pm 0.0$ & $27.3 \pm 0.1$ \\
\hline $18: 3 n-3$ & $4.3 \pm 0.0$ & $4.1 \pm 0.0$ & $4.4 \pm 0.0$ & $4.8 \pm 0.0$ & $4.8 \pm 0.0$ & $5.4 \pm 0.0$ \\
\hline $18: 4 n-3$ & $1.5 \pm 0.0$ & $1.9 \pm 0.0$ & $1.5 \pm 0.0$ & $1.2 \pm 0.0$ & $1.5 \pm 0.0$ & $1.0 \pm 0.0$ \\
\hline $20: 5 n-3$ & $6.4 \pm 0.1$ & $7.8 \pm 0.2$ & $7.3 \pm 0.1$ & $6.8 \pm 0.1$ & $6.2 \pm 0.0$ & $4.2 \pm 0.1$ \\
\hline $22: 5 n-3$ & $1.2 \pm 0.0$ & $1.5 \pm 0.1$ & $1.2 \pm 0.1$ & $0.9 \pm 0.0$ & $1.2 \pm 0.0$ & $0.8 \pm 0.0$ \\
\hline $22: 6 n-3$ & $6.9 \pm 0.2$ & $7.8 \pm 0.2$ & $8.4 \pm 0.2$ & $9.3 \pm 0.2$ & $6.3 \pm 0.0$ & $4.2 \pm 0.1$ \\
\hline$n-3^{d}$ & $21.2 \pm 0.4$ & $24.2 \pm 0.3$ & $23.8 \pm 0.5$ & $23.8 \pm 0.4$ & $20.8 \pm 0.1$ & $16.1 \pm 0.2$ \\
\hline$n-3 / n-6$ & $1.4 \pm 0.0$ & $1.7 \pm 0.0$ & $1.6 \pm 0.0$ & $1.5 \pm 0.0$ & $1.0 \pm 0.0$ & $0.6 \pm 0.0$ \\
\hline PUFA $^{e}$ & $37.7 \pm 0.4$ & $40.5 \pm 0.4$ & $39.9 \pm 0.5$ & $40.9 \pm 0.4$ & $42.8 \pm 0.1$ & $44.4 \pm 0.3$ \\
\hline MC-PUFA & $20.1 \pm 0.1$ & $19.7 \pm 0.0$ & $19.8 \pm 0.0$ & $21.1 \pm 0.1$ & $26.2 \pm 0.1$ & $33.3 \pm 0.1$ \\
\hline LC-PUFA & $16.2 \pm 0.4$ & $19.0 \pm 0.4$ & $18.6 \pm 0.4$ & $18.6 \pm 0.3$ & $15.2 \pm 0.1$ & $10.1 \pm 0.2$ \\
\hline
\end{tabular}

a Saturated fatty acids-sum of all fatty acids without double bonds; includes 12:0, 13:0, 15:0, 17:0, 20:0, and 24:0 in addition to individually reported SFA

b Monounsaturated fatty acids - sum of all fatty acids with a single double bond; includes 14:1, 20:1n-9 and 24:1n-9

c Sum of all $n-6$ fatty acids; includes 18:3n-6, 20:2n-6, and 20:3n-6

d Sum of all n-3 fatty acids; includes 20:3n-3 and 20:4n-3

e Polyunsaturated fatty acids - sum of all fatty acids with $\geq 2$ double bonds; includes $18: 3 n-4,18: 3 n-6,20: 2 n-6,20: 3 n-6,20: 3 n-3$, and 20:4n-3

f Medium-chain PUFA - sum of all PUFA with chain length of 18 carbon atoms; includes 18:3n-4 and 18:3n-6

$\mathrm{g}$ Long-chain PUFA-sum of all fatty acids with chain length $\geq 20$ carbon atoms and double bonds $\geq 3$; includes 20:3n-6, 20:3n-3, and 20:4n-3

and polar lipid fractions (Tables 3, 4). Reducing fish meal from 30 to $10 \%$ of the formulation necessitated the inclusion of additional oil to maintain total dietary lipid content. Greater inclusion of neutral lipid-rich fish oil thus increased the level of 20:5n-3 (eicosapentaenoic acid), 22:6n-3, and total long-chain polyunsaturated fatty acids (LC-PUFA) within the neutral lipid fraction of the $10 \%$ FM feed compared to the $30 \%$ FM feed. By replacing fish oil, lecithin supplementation progressively reduced the contribution of fish oil to neutral lipid and LC-PUFA within this lipid fraction. Sparing fish meal with alternative feedstuffs had the opposite effect on the prevalence of marine-associated fatty acids within the polar lipid fraction. Although the $10 \% \mathrm{FM}$ formulation was amended with fish oil to balance total lipid content, phospholipids were not supplemented and thus the polar lipid fraction reflected only the residual phospholipids contributed by the plant-origin feedstuffs and remaining fish meal. As a result, the LC-PUFA-rich polar fraction in the 30\% FM feed was largely replaced by an 18:2n-6 (linoleic acid)-rich polar lipid fraction in the $10 \%$ FM feed. Supplementation with marine lecithin corrected for this effect by progressively increasing the amount polar lipid-bound LC-PUFA, whereas supplementation with soy lecithin maintained an 18:2n-6-rich polar lipid profile.

Fish fed the $30 \%$ FM control feed exhibited feed intake $(2.51 \%$ body weight $/$ day), growth (weight gain $=272 \%$, SGR $=2.12 \%$ body weight/day), and a feed conversion ratio (1.10) consistent with juvenile hybrid striped bass cultured under near-optimal conditions in recirculation systems (Table 5). Although statistical significance was not resolved, fish fed the 10\% FM diet exhibited numerically inferior performance compared to the $30 \% \mathrm{FM}$ control group. Though significant differences were not observed for all parameters or dietary treatment groups, fish fed the amended $10 \%$ FM diets generally exhibited performance greater than the $10 \% \mathrm{FM}$ treatment group. This effect was particularly evident with the $10 \% \mathrm{FM}+1 \mathrm{X}$ Marine $\mathrm{PL}$ and $10 \% \mathrm{FM}+2 \mathrm{X}$ Marine PL feeds, which yielded significantly greater feed intake, weight gain, SGR, and TGC than both the 10\% FM and 30\% FM feeds. Hepatosomatic index was significantly lower among fish fed the $10 \% \mathrm{FM}$ diets. Liposomatic index was equivalent among fish fed the $30 \%$ FM and $10 \%$ FM feeds, and these values were significantly lower than those associated with all other treatments except the $10 \% \mathrm{FM}+1 \mathrm{X}$ Soy PL (which yielded a numerically, but not significantly greater LSI than the 30\% FM and 10\% FM feeds). No mortalities occurred, and all fish appeared healthy and demonstrated normal behavior throughout the trial.

\section{Discussion}

Previous research evaluating alternative proteins in hybrid striped bass feeds has indicated that aggressive fish meal sparing (e.g., reducing fish meal inclusion below 10\%) or complete replacement can result in reduced growth performance, often 
Table 3 Fatty acid composition (\% fatty acid methyl esters [FAME]) of dietary neutral lipid fractions. Values represent LS-means \pm SEM of triplicate samples.

\begin{tabular}{|c|c|c|c|c|c|c|}
\hline Fatty Acid(s) ${ }^{\mathrm{a}}$ & $30 \% \mathrm{FM}$ & $10 \% \mathrm{FM}$ & $10 \% \mathrm{FM}+1 \mathrm{X}$ Marine PL & $10 \% \mathrm{FM}+2 \times$ Marine PL & $10 \% \mathrm{FM}+1 \mathrm{X}$ Soy $\mathrm{PL}$ & $10 \% \mathrm{FM}+2 \times$ Soy $\mathrm{PL}$ \\
\hline 14:0 & $4.3 \pm 0.0$ & $5.3 \pm 0.0$ & $4.9 \pm 0.0$ & $4.1 \pm 0.1$ & $4.7 \pm 0.0$ & $3.5 \pm 0.0$ \\
\hline $16: 0$ & $12.4 \pm 0.0$ & $13.8 \pm 0.1$ & $13.1 \pm 0.1$ & $11.8 \pm 0.1$ & $13.3 \pm 0.1$ & $11.9 \pm 0.1$ \\
\hline 18:0 & $2.9 \pm 0.0$ & $3.1 \pm 0.0$ & $3.0 \pm 0.0$ & $2.8 \pm 0.0$ & $3.2 \pm 0.0$ & $3.2 \pm 0.0$ \\
\hline SFA & $21.2 \pm 0.0$ & $23.7 \pm 0.1$ & $22.4 \pm 0.0$ & $20.2 \pm 0.2$ & $22.8 \pm 0.2$ & $20.0 \pm 0.1$ \\
\hline $16: 1 n-7$ & $5.4 \pm 0.0$ & $6.8 \pm 0.0$ & $6.3 \pm 0.0$ & $5.2 \pm 0.1$ & $6.0 \pm 0.0$ & $4.4 \pm 0.0$ \\
\hline $18: 1 n-7$ & $3.0 \pm 0.0$ & $3.0 \pm 0.0$ & $3.1 \pm 0.0$ & $3.2 \pm 0.0$ & $3.0 \pm 0.0$ & $2.8 \pm 0.0$ \\
\hline $18: 1 n-9$ & $32.1 \pm 0.2$ & $25.6 \pm 0.1$ & $29.0 \pm 0.2$ & $34.1 \pm 0.3$ & $28.8 \pm 0.3$ & $32.6 \pm 0.1$ \\
\hline $20: 1 n-9$ & $1.0 \pm 0.0$ & $1.0 \pm 0.0$ & $1.2 \pm 0.1$ & $1.1 \pm 0.0$ & $1.0 \pm 0.0$ & $0.9 \pm 0.0$ \\
\hline MUFA & $41.4 \pm 0.3$ & $36.5 \pm 0.1$ & $39.6 \pm 0.0$ & $43.5 \pm 0.4$ & $38.9 \pm 0.4$ & $40.7 \pm 0.1$ \\
\hline $18: 2 n-6$ & $13.2 \pm 0.1$ & $10.7 \pm 0.0$ & $10.8 \pm 0.0$ & $12.7 \pm 0.0$ & $14.1 \pm 0.0$ & $19.2 \pm 0.0$ \\
\hline $20: 4 n-6$ & $0.6 \pm 0.0$ & $0.7 \pm 0.0$ & $0.6 \pm 0.0$ & $0.5 \pm 0.0$ & $0.6 \pm 0.0$ & $0.4 \pm 0.0$ \\
\hline$n-6$ & $14.3 \pm 0.1$ & $12.0 \pm 0.0$ & $12.0 \pm 0.0$ & $13.6 \pm 0.1$ & $15.2 \pm 0.0$ & $19.9 \pm 0.0$ \\
\hline $18: 3 n-3$ & $4.5 \pm 0.0$ & $4.0 \pm 0.0$ & $4.6 \pm 0.0$ & $5.3 \pm 0.1$ & $4.4 \pm 0.0$ & $5.1 \pm 0.0$ \\
\hline $18: 4 n-3$ & $1.6 \pm 0.0$ & $2.0 \pm 0.0$ & $1.8 \pm 0.0$ & $1.5 \pm 0.0$ & $1.6 \pm 0.0$ & $1.2 \pm 0.0$ \\
\hline $20: 5 n-3$ & $6.6 \pm 0.0$ & $8.4 \pm 0.1$ & $7.6 \pm 0.1$ & $6.2 \pm 0.2$ & $6.7 \pm 0.2$ & $5.1 \pm 0.1$ \\
\hline $22: 5 n-3$ & $1.3 \pm 0.0$ & $1.7 \pm 0.0$ & $1.5 \pm 0.0$ & $1.2 \pm 0.0$ & $1.4 \pm 0.1$ & $1.0 \pm 0.0$ \\
\hline $22: 6 n-3$ & $6.8 \pm 0.1$ & $8.6 \pm 0.1$ & $7.8 \pm 0.2$ & $6.4 \pm 0.2$ & $6.7 \pm 0.3$ & $5.1 \pm 0.1$ \\
\hline$n-3$ & $21.7 \pm 0.2$ & $26.0 \pm 0.2$ & $24.4 \pm 0.4$ & $21.3 \pm 0.6$ & $21.6 \pm 0.6$ & $18.2 \pm 0.2$ \\
\hline$n-3 / n-6$ & $1.5 \pm 0.0$ & $2.2 \pm 0.0$ & $2.0 \pm 0.0$ & $1.6 \pm 0.0$ & $1.4 \pm 0.0$ & $0.9 \pm 0.0$ \\
\hline PUFA & $37.4 \pm 0.3$ & $39.8 \pm 0.2$ & $38.0 \pm 0.4$ & $36.3 \pm 0.6$ & $38.3 \pm 0.6$ & $39.3 \pm 0.2$ \\
\hline MC-PUFA & $19.7 \pm 0.1$ & $17.2 \pm 0.0$ & $17.6 \pm 0.0$ & $19.8 \pm 0.1$ & $20.5 \pm 0.1$ & $25.8 \pm 0.0$ \\
\hline LC-PUFA & $16.3 \pm 0.2$ & $20.8 \pm 0.2$ & $18.8 \pm 0.3$ & $15.2 \pm 0.5$ & $16.3 \pm 0.5$ & $12.3 \pm 0.2$ \\
\hline
\end{tabular}

${ }^{\text {a }}$ All abbreviations and groupings are as defined in Table 2 .

Table 4 Fatty acid composition (\% fatty acid methyl esters [FAME]) of dietary polar lipid fractions. Values represent means of triplicate samples, except where noted.

\begin{tabular}{|c|c|c|c|c|c|c|}
\hline Fatty Acid(s) ${ }^{\mathrm{a}}$ & $30 \% \mathrm{FM}^{\mathrm{b}}$ & $10 \% \mathrm{FM}^{\mathrm{b}}$ & 10\% FM + 1X Marine PL & $10 \% \mathrm{FM}+2 \mathrm{X}$ Marine PL & $10 \% \mathrm{FM}+1 \mathrm{X}$ Soy $\mathrm{PL}$ & $10 \% \mathrm{FM}+2 \times$ Soy $\mathrm{PL}$ \\
\hline $14: 0$ & 1.1 & 0.6 & $0.9 \pm 0.0$ & $1.1 \pm 0.0$ & $0.3 \pm 0.0$ & $0.2 \pm 0.0$ \\
\hline $16: 0$ & 21.2 & 16.6 & $24.0 \pm 0.1$ & $26.7 \pm 0.2$ & $16.7 \pm 0.1$ & $16.6 \pm 0.0$ \\
\hline 18:0 & 4.7 & 4 & $3.3 \pm 0.2$ & $3.0 \pm 0.0$ & $3.7 \pm 0.0$ & $3.5 \pm 0.0$ \\
\hline $\mathrm{SF}^{\mathrm{A}}$ & 34.2 & 26.2 & $23.0 \pm 0.0$ & $33.0 \pm 0.1$ & $24.3 \pm 0.2$ & $23.0 \pm 0.0$ \\
\hline $16: 1 n-7$ & 1.7 & 0.9 & $0.5 \pm 0.0$ & $0.5 \pm 0.0$ & $0.5 \pm 0.0$ & $0.4 \pm 0.0$ \\
\hline $18: 1 n-7$ & 1.7 & 1.6 & $1.4 \pm 0.0$ & $1.3 \pm 0.0$ & $1.5 \pm 0.0$ & $1.4 \pm 0.0$ \\
\hline $18: 1 n-9$ & 7.1 & 6.6 & $4.8 \pm 0.0$ & $4.2 \pm 0.1$ & $8.2 \pm 0.1$ & $9.0 \pm 0.0$ \\
\hline $20: 1 n-9$ & 0.2 & 0.1 & $1.2 \pm 0.0$ & $1.6 \pm 0.0$ & $0.0 \pm 0.0$ & $0.0 \pm 0.0$ \\
\hline MUFA & 10.8 & 9.3 & $16.1 \pm 0.0$ & $7.7 \pm 0.1$ & $10.1 \pm 0.1$ & $10.7 \pm 0.1$ \\
\hline $18: 2 n-6$ & 28.2 & 49.1 & $31.1 \pm 0.2$ & $22.7 \pm 0.2$ & $54.4 \pm 0.2$ & $56.4 \pm 0.1$ \\
\hline $20: 4 n-6$ & 1.3 & 0.4 & $1.0 \pm 0.0$ & $1.2 \pm 0.0$ & $0.2 \pm 0.0$ & $0.2 \pm 0.0$ \\
\hline$n-6$ & 29.7 & 49.7 & $31.3 \pm 0.2$ & $24.1 \pm 0.1$ & $54.7 \pm 0.2$ & $56.5 \pm 0.1$ \\
\hline $18: 3 n-3$ & 4.1 & 7.5 & $4.5 \pm 0.0$ & $3.3 \pm 0.0$ & $7.4 \pm 0.0$ & $7.3 \pm 0.0$ \\
\hline $18: 4 n-3$ & 0.3 & 0.1 & $0.1 \pm 0.0$ & $0.1 \pm 0.0$ & $0.0 \pm 0.0$ & $0.0 \pm 0.0$ \\
\hline $20: 5 n-3$ & 5.7 & 2.1 & $6.5 \pm 0.0$ & $8.3 \pm 0.0$ & $1.0 \pm 0.0$ & $0.7 \pm 0.0$ \\
\hline $22: 5 n-3$ & 0.8 & 0.3 & $0.4 \pm 0.0$ & $0.4 \pm 0.0$ & $0.2 \pm 0.0$ & $0.0 \pm 0.0$ \\
\hline $22: 6 n-3$ & 14.1 & 4.8 & $18.0 \pm 0.1$ & $23.0 \pm 0.1$ & $2.3 \pm 0.0$ & $1.6 \pm 0.0$ \\
\hline$n-3$ & 25.3 & 14.9 & $19.9 \pm 0.1$ & $35.3 \pm 0.2$ & $10.9 \pm 0.0$ & $9.7 \pm 0.0$ \\
\hline$n-3 / n-6$ & 0.9 & 0.3 & $0.6 \pm 0.0$ & $1.5 \pm 0.0$ & $0.2 \pm 0.0$ & $0.2 \pm 0.0$ \\
\hline PUFA & 55 & 64.5 & $53.0 \pm 0.2$ & $59.3 \pm 0.2$ & $65.6 \pm 0.2$ & $66.3 \pm 0.1$ \\
\hline MC-PUFA & 32.6 & 56.7 & $32.4 \pm 0.2$ & $26.2 \pm 0.1$ & $61.8 \pm 0.2$ & $63.7 \pm 0.1$ \\
\hline LC-PUFA & 22.2 & 7.7 & $25.9 \pm 0.1$ & $33.0 \pm 0.2$ & $3.7 \pm 0.0$ & $2.5 \pm 0.0$ \\
\hline
\end{tabular}

${ }^{\text {a }}$ All abbreviations and groupings are as defined in Table 2 .

${ }^{b}$ Only a single replicate was successfully analyzed; remaining replicates were lost 
as a consequence of reduced feed palatability and intake $[6,19,20,21,22]$. In our previous research, we have determined that $10 \%$ fish meal is the approximate threshold at which fish meal sparing begins to impact palatability and growth performance in juvenile hybrid striped bass: although statistically significant impairment was not observed in each of these studies, $10 \%$ fish meal appears to be a relatively conservative estimate of the minimum appropriate inclusion level for this taxon $[22,24,25]$. Our present results are consistent with the concept of a $10 \%$ fish meal inclusion threshold: whereas statistically significant impairment was not observed, fish fed the $10 \%$ FM feed exhibited numerically lower weight gain and SGR compared to those fed the $30 \%$ FM feed despite numerically higher feed intake of the $10 \%$ FM feed. It is somewhat unsurprising that the addition of nutrients which may be limiting in the $10 \%$ FM feed, specifically phospholipids, was generally associated with improvements in growth performance. What is surprising, however, is that certain of these amendments, most obviously marine lecithin, resulted in significant improvement over the $30 \%$ FM feed as well as the $10 \%$ FM feed.

Previous research has indicated that supplementation with lecithin can improve intake, digestibility, and utilization of aquafeeds, particularly those containing little-to-no fish meal. Although a preliminary study failed to demonstrate growth promotion [26], Iwashita et al. [9] reported supplementing fish meal-free rainbow trout diets with soy lecithin improved fish growth and feed intake, and also corrected for some histological abnormalities observed within the gut of fish fed the un-supplemented, fish meal-free diet. Similar results were also achieved with bovine gall powder and cholyltaurine supplements, though these products appeared to be more effective in maintaining gastrointestinal integrity and promoting growth than soy lecithin [9]. These authors concluded that the growth promoting effects of these supplements were associated with somewhat distinct functions: whereas gall powder (containing phospholipids, deoxycholyltaurine, and other proteins) and synthetic cholyltaurine act primarily via maintenance of gut integrity and stimulation of digestive and metabolic activities, lecithin acts primarily by increasing feed intake [9]. Growth of Japanese flounder [10] and amberjack [11] fed fish meal-free diets was progressively improved by amending the diets with $2-4 \%$ soy lecithin, and the authors also attributed these effects to greater acceptance and intake of the lecithinsupplemented feeds. In a series of studies evaluating dietary inclusion of lecithin and soy protein in salmonid feeds, Poston $[8,12,13,27]$ reported similar increases in feed intake and growth performance using lecithin as a supplement in feeds for rainbow trout and Atlantic salmon. Our results are consistent with these accounts, suggesting improved performance among fish fed the lecithin-amended feeds is due, in part, to increased feed intake. Although Sealey et al. [28] reported hybrid striped bass growth was unaffected by dietary supplementation with soy lecithin, the feeds used in this study contained approximately $60 \%$ fish meal (containing $2.6 \%$ lipid) and approximately $7 \%$ Fish Oil and were likely to have contained sufficient amounts of polar lipid as to preclude performance enhancement through additional supplementation.

In their studies of phospholipid supplementation in yellowtail Seriola quinqueradiata and gilthead seabream Sparus aurata, respectively, Harada [29] and Koven et al. [30] suggested the trimethyl group associated with the nitrogen moiety of phosphatidylcholine (the primary phospholipid associated with lecithins) could act as an attractant in fish feeds. The proposed attractant properties of phosphatidylcholine do not explain the differential results achieved with marine vs. soy lecithin in the present study or our previous work with these supplements in cobia Rachycentron canadum feeds [24]. As in the present study,

Table 5 Growth performance of hybrid striped bass by dietary treatment. Values are presented as LS-means \pm pooled SE, and P vales associated with one-way ANOVA tests are provided. Means with common letter labels are not significantly different, $(p>0.05)$.

\begin{tabular}{|c|c|c|c|c|c|c|c|c|}
\hline Parameter & $30 \% \mathrm{FM}$ & $10 \%$ FM & $\begin{array}{c}10 \% \mathrm{FM}+1 \mathrm{X} \\
\text { Marine PL }\end{array}$ & $\begin{array}{c}10 \% \text { FM }+2 X \\
\text { Marine PL }\end{array}$ & $\begin{array}{c}10 \% \mathrm{FM}+1 \mathrm{X} \\
\text { Soy PL }\end{array}$ & $\begin{array}{l}10 \% \text { FM }+2 X \\
\text { Soy PL }\end{array}$ & Pooled SE & p-value \\
\hline Survival & 100 & 100 & 100 & 100 & 100 & 100 & --- & --- \\
\hline Initial weight (g) & 33.4 & 33.3 & 33.1 & 33.7 & 33.2 & 32.4 & 0.8 & 0.743 \\
\hline Final weight (g) & $124.2 x$ & $117.5 x$ & $142.9 \mathrm{yz}$ & $150.1 \mathrm{z}$ & $128.5 x y$ & 129.6 xy & 5.5 & $<0.001$ \\
\hline Weight gain (\%) & $272 w x$ & $252 w$ & $332 \mathrm{zy}$ & $345 z$ & $287 w x$ & $300 x y$ & 13 & $<0.001$ \\
\hline $\begin{array}{l}\text { Cumulative individual } \\
\text { consumption }\end{array}$ & $100 v$ & $106 \mathrm{vw}$ & $126 y z$ & $131 z$ & $113 w x$ & $117 x y$ & 3 & $<0.001$ \\
\hline (g, dry matter) & & & & & & & & \\
\hline Feed conversion ratio & 1.10 & 1.27 & 1.15 & 1.13 & 1.19 & 1.22 & 0.06 & 0.142 \\
\hline Specific growth rate & $2.12 w x$ & $2.03 w$ & $2.36 \mathrm{yz}$ & $2.41 z$ & $2.18 w x y$ & $2.23 x y z$ & 0.06 & $<0.001$ \\
\hline (\% body weight/day) & & & & & & & & \\
\hline $\begin{array}{c}\text { Feed intake } \\
\text { (\% body weight/day) }\end{array}$ & $2.51 \mathrm{y}$ & $2.74 y z$ & $2.96 z$ & $2.98 z$ & $2.79 \mathrm{yz}$ & $2.94 \mathrm{z}$ & 0.12 & 0.006 \\
\hline $\begin{array}{c}\text { Thermal Growth } \\
\text { Coefficient }\end{array}$ & $1.17 \mathrm{x}$ & $1.11 \mathrm{x}$ & $1.33 \mathrm{yz}$ & $1.39 \mathrm{z}$ & $1.21 \mathrm{xy}$ & $1.22 x y$ & 0.05 & $<0.001$ \\
\hline Hepatosomatic Index & $1.78 z$ & $1.25 \mathrm{y}$ & $1.33 y$ & $1.31 \mathrm{y}$ & $1.16 y$ & $1.30 \mathrm{y}$ & 0.08 & $<0.001$ \\
\hline Liposomatic Index & $2.42 x$ & $2.27 x$ & $3.21 \mathrm{yz}$ & $3.58 z$ & $2.67 x y$ & $3.38 \mathrm{yz}$ & 0.25 & $<0.001$ \\
\hline
\end{tabular}


supplementing reduced fish meal cobia feeds with soy lecithin did not elicit the same magnitude of growth promotion as did supplementation with marine lecithin [24]. Although the diets were formulated to be replete with all nutrients known to be required for optimal growth in both studies, marine lecithin supplementation conferred greater benefit to the reduced fish meal feeds than equivalent supplementation with soy lecithin. This would seem to suggest a distinct functional property, not the potential attractant characteristics of a trimethyl group common in both phosphatidylcholine-rich lecithin products, is responsible for the particularly beneficial effects of supplementing the diet with marine lecithin. The primary difference between the two lecithin products is the fatty acid composition of the phospholipids they contain: the soy lecithin is particularly rich in $18: 2 n-6$, whereas $22: 6 n-3$ is abundant in the marine lecithin product. Previous research has demonstrated that provision of essential and physiologically relevant fatty acids like 22:6n3 is critical for normal growth and development of many fishes [31], including hybrid striped bass [32], and that these nutrients are more potent when provided in the context of polar rather than neutral lipid $[7,14,15]$. Thus, a growth-promoting effect of providing additional phospholipid-bound $22: 6 n-3$ vs. $18: 2 n-6$ seems a plausible explanation of the present results. However, it is not clear what the underlying mechanism responsible for these differential effects might be.

In conclusion, it appears that amending reduced fish meal feeds with phospholipids can improve feed intake and growth in hybrid striped bass, and supplementing the diet with marine lecithin in particular may increase hybrid striped bass growth beyond that typically achieved with fish meal-based formulations. Of course, the incorporation of marine lecithin as a feed ingredient is counter to the rationale for fish meal sparing, particularly the arguments related to economic and sustainability concerns regarding fish meal. Although sustainability may be less of a concern for marine lecithin than for other marine-derived ingredients (marine lecithin is derived from processing wastes generated by industrialized marine fisheries), it will almost certainly be more expensive that soy lecithin, now and in the future. Whether the added cost of marine lecithin supplementation is justified by the apparent benefits of this type of ingredient will depend upon the circumstances at hand, e.g., the feed formulation, intended fish taxon, feedstuff pricing. Further research is recommended to further elucidate the potentially interrelated functions of phospholipids and the fatty acids they carry in determining feed acceptance and growth performance in cultured fish. 


\section{References}

1 Gatlin DM, Barrows FT, Brown P, Dabrowski K, Gaylord TG, et al. (2007) Expanding the utilization of sustainable plant products in aquafeeds: a review. Aquaculture Research 38: 551-579.

2 Thompson KR, Rawles SD, Metts LS, Smith R, Wimsatt A, et al. (2008) Digestibility of dry matter, protein, lipid, and organic matter of two fish meals, two poultry by-product meals, soybean meal, and distiller's dried grains with soluble in practical diets for sunshine bass, Morone chrysops $\times$ M. saxatilis. Journal of the World Aquaculture Society 39: 352-363.

3 Rawles SD, Gatlin DM (2000) Nutrient digestibility of common feedstuffs in extruded diets for sunshine bass Morone chrysops $\times M$. saxatilis. Journal of the World Aquaculture Society 31: 570-579.

4 Brown PB, Wilson KA, Hodgin Y, Stanley JD (1997) Use of soy protein concentrates \& lecithin products in diets fed to coho and Atlantic salmon. Journal of the American Oil Chemists Society 74: 187-193.

5 Keembiyehetty CN, Gatlin DM (1997) Performance of sunshine bass fed soybean-meal-based diets supplemented with different methionine compounds. The Progressive Fish-Culturist 59: 28-30.

6 Laporte J, Trushenski J (2012) Production performance, stress tolerance, and intestinal integrity of sunshine bass fed increasing levels of soybean meal. Journal of Animal Physiology and Animal Nutrition 96: 513-526.

7 Tocher DR, Bendiksen EÅ, Campbell PJ, Bell JG (2008) The role of phospholipids in nutrition and metabolism of teleost fish. Aquaculture 280: 21-34.

8 Poston HA (1991b) Response of rainbow trout to soy lecithin, choline, and autoclaved isolated soy protein. The Progressive Fish Culturist 53: 85-90.

9 Iwashita Y, Suzuki N, Yamamoto T, Shibata JI, Isokawa K, et al. (2008) Supplemental effect of cholyltaurine and soybean lecithin to a soybean meal-based fish meal-free diet on hepatic and intestinal morphology of rainbow trout Oncorhynchus mykiss. Fisheries Sciences 74: 1083-1095.

10 Uyan O, Koshio S, Ishikawa M, Uyan S, Ren T, et al. (2007) Effects of dietary phosphorus and phospholipid level on growth, and phosphorus deficiency signs in juvenile Japanese flounder, Paralichthys olivaceus. Aquaculture 267: 44-54.

11 Uyan O, Koshio S, Ishikawa M, Yokoyama S, Uyan S, et al. (2009) The influence of dietary phospholipid level on the performances of juvenile amberjack, Seriola dumerili, fed non-fishmeal diets. Aquaculture Nutrition 15: 550-557.

12 Poston HA (1990) Effect of body size on growth, survival, and chemical composition of Atlantic salmon fed soy lecithin and choline. The Progressive Fish Culturist 52: 226-230.

13 Poston HA (1990) Performance of rainbow trout fry fed supplemental soy lecithin and choline. The Progressive Fish Culturist 52: 218-225.

14 Cotteau P, Geurden I, Camara MR, Bergot P, Sorgeloos P, et al. (1997) Review on the dietary effects of phospholipids in fish and crustacean larviculture. Aquaculture 155: 149-164.

15 Villeneuve L, Gisbert E, Zambonino-Infante JL, Quazuguel P, Cahu CL, et al. (2005) Effect of nature of dietary lipids on European sea bass morphogenesis: implication of retinoid receptors. British Journal of Nutrition 94: 877-884.

16 Folch J, Lees M, Sloane-Stanley GH (1957) A simple method for the isolation and purification of total lipids from animal tissues. Journal of Biological Chemistry 226: 497-509.
17 Laporte J, Trushenski JT (2011) Growth performance and tissue fatty acid composition of largemouth bass fed diets containing fish oil or blends of fish oil and soy-derived lipids. North American Journal of Aquaculture 73: 435-444.

18 Kaluzny MA, Duncan LA, Merritt MV, Epps DE (1985) Rapid separation of lipid classes in high yield and purity using bonded phase columns. Journal of Lipid Research 26: 135-140.

19 Lewis HA, Kohler CC (2008) Corn gluten meal partially replaces dietary fish meal without compromising growth or fatty acid composition of sunshine bass. North American Journal of Aquaculture 70: 50-60.

20 Gause BR, Trushenski JT (2011a.) Replacement of fish meal with ethanol yeast in the diets of sunshine bass Morone chrysops $\hat{\sigma} \times M$. saxatilis 9 . North American Journal of Aquaculture 73: 97-103.

21 Gause BR, Trushenski JT (2011b) Production performance and stress tolerance of sunshine bass raised on reduced fish meal feeds containing ethanol yeast. North American Journal of Aquaculture 73: 168-175.

22 Blaufuss P, Trushenski JT (2011) Exploring soy-derived alternatives to fish meal: using soy protein concentrate and soy protein isolate in hybrid striped bass (Morone chrysops $\times$ M. saxatilis) feeds. North American Journal of Aquaculture 74: 8-19.

23 Hill HA, Trushenski JT, Kohler CC (2013) Utilization of soluble canola protein concentrate as an attractant enhances production performance of sunshine bass fed reduced fish meal, plant-based diets. Journal of the World Aquaculture Society 44: 124-132.

24 Trushenski JT, Gause BR (2013) Comparative value of fish meal alternatives as protein sources in feeds for hybrid striped bass. North American Journal of Aquaculture 75: 329-341.

25 Trushenski J, Schwarz M, Pessoa WVN, Mulligan B, Crouse C, et al. (2013) Amending reduced fish-meal feeds with marine lecithin, but not soy lecithin, improves the growth of juvenile cobia and may attenuate heighted responses to stress challenge. Journal of Animal Physiology and Animal Nutrition 97: 170-180.

26 Suzuki N, Yamamoto T (2004) Histological observations of intestinal degeneration of defatted soybean meal diet and supplemental effect of soybean lecithin for fingerling rainbow trout, Oncorhynchus mykiss. Journal of the School of Marine Science and Technology 2: 25-36.

27 Poston HA (1991a) Response of Atlantic salmon fry to feed-grade lecithin and choline. The Progressive Fish Culturist 53: 224-228.

28 Sealey WM, Craig SR, Gatlin DM (2001) Dietary cholesterol and lecithin have limited effects on growth and body composition of hybrid striped bass (Morone chrysops $\times$ M. saxatilis). Aquaculture Nutrition 7: 25-31.

29 Harada K (1987) Relationships between structure and feeding attraction activity of certain L-amino acids and lecithin in aquatic animals. Bulletin of the Japanese Society of Scientific Fisheries 53: 2243-2247.

30 Koven WM, Tandler A, Sklan D, Kissil GW (1993) The association of eicosapentaenoic and docosahexaenoic acids in the main phospholipids of different-age Sparus aurata larvae with growth. Aquaculture 116: 71-82.

31 Sargent J, Bell G, McEvoy L, Tocher D, Estevez A, et al. (1999) Recent developments in the essential fatty acid nutrition of fish. Aquaculture 177: 191-199.

32 Nematipour GR, Gatlin DM (1993) Effects of different kinds of dietary lipid on growth and fatty acid composition of juvenile sunshine bass, Morone chrysops x M. saxatilis. Aquaculture 114: 141-154. 